UNIQUE APPLICATIONS OF \section{OPTICAL PROPERTIES OF SILICON NANOSTRUCTURES}

YURY V. RYABCHIKOV

HiLASE Centre, Institute of Physics of the Czech Academy of Sciences, Dolni Brezany, Czech Republic

DOI : 10.17973/MMSJ.2019_12_2019102

e-mail: yury.ryabchikov@hilase.cz

Silicon nanostructures can be prepared by different methods that considerably change their optical properties depending on experimental conditions. They are also very sensitive to molecular environment and external influences that can be used for multiple applications in different areas. Silicon nanostructures prepared by laser ablation show large perspectives for molecular sensing and biomedical applications. In this paper, an overview of optical properties of silicon nanostructures with focus on nanoparticles prepared by ultrafast laser ablation in liquids is provided. It is shown that they reveal wide prospects for applications in nanobiomedicine and their unique characteristics can be significantly enhanced due to laser-induced metal incorporation. Metal inclusions lead to appearance of plasmonic properties in semiconductor nanomaterials that can be applied for molecule detection using surface enhancing of optical response.

KEYWORDS

silicon nanoparticles, laser ablation, porous silicon, optical properties, photoluminescence, biological application

\section{INTRODUCTION}

Silicon is one of the most widespread elements on the Earth that is widely used in microelectronics and photovoltaics [Priolo 2014; Yue 2014; Govoni 2012; Vetterl 2000; Yates 1998; Clemens 1997]. Nevertheless, it cannot be applied for optoelectronic purposes due to its poor emission properties. However, they are found to be considerably changed due to silicon nanostructuring in consequence of significant confinement of mobility of charge carriers. Indeed, electrochemical etching of silicon wafers leads to formation of a highly porous structure containing nanocrystals with sizes less than $5 \mathrm{~nm}$. Such a small size accompanied with strong quantum confinement effect provokes effective emission from the nanoscrystals in orange-red spectral range under UV-visible excitation [Cullis 1997; Brus 1994; Quin1993; Pavesi 1993; Heinrich 1992; Tischler 1992; Xie 1992]. Subsequent mechanical milling of prepared porous silicon layers allows significant reduction of their size forming porous silicon-based nanoparticles (PS-Si NPs).

At the same time, pulsed laser ablation of silicon wafers immersed in liquids yields silicon nanoparticles (LA-Si NPs) of several tens nm [Ryabchikov 2019a; Intartaglia 2011; Kuzmin 2010; Semaltianos 2010; Rioux 2009] beyond quantum confinement effect that leads to absence of linear-excited emission properties in silicon nanostructures. So, the choice of a synthesis method significantly influences properties of formed nanostructures.

In this paper, silicon nanostructures are prepared by two different methods of treatment of silicon wafers in liquid environment using either electrochemical etching or pulsed laser irradiation. A comparative analysis of their optical properties is carried out. Influence of adsorption of different gases on photoluminescence (PL) properties of electrochemically prepared silicon nanostructures and sensitisation of singlet oxygen generation are investigated. Linear and nonlinear optical properties of laser synthesized silicon nanoparticles are studied. Their size change due to dissolution in the physiological medium as well as due to structural modification in the presence of gold is detected. Capability of molecule detection using Rhodamine B dye molecules by Si-based NPs is proofed.

\section{EXPERIMENTAL METHODS}

Silicon nanoparticles are prepared by two methods: (i) electrochemical etching and (ii) laser ablation of a silicon wafer (Fig. 1) in a liquid environment. In the first case, chemical processes of silicon etching occur in the $\mathrm{HF}: \mathrm{C}_{2} \mathrm{H}_{5} \mathrm{OH}$ solution (1:1 volume ratio) at $50 \mathrm{~mA} / \mathrm{cm}^{2}$ current density. In the second case, formation of nanoparticles takes places in deionized water induced by action of a femtosecond laser $(800 \mathrm{~nm}, 130$ fs, $100 \mu \mathrm{J} /$ pulse, $1000 \mathrm{~Hz}$ ). In order to form composite silicongold nanoparticles (Si@Au NPs), previously formed laserablated Si NPs are structurally modified due to laser ablation of

\section{Electrochemical etching Laser ablation}

$$
\mathrm{HF}(48 \%): \mathrm{C}_{2} \mathrm{H}_{5} \mathrm{OH}=1: 1 \quad \text { Laser irradiation }
$$

\section{Laser irradiation}

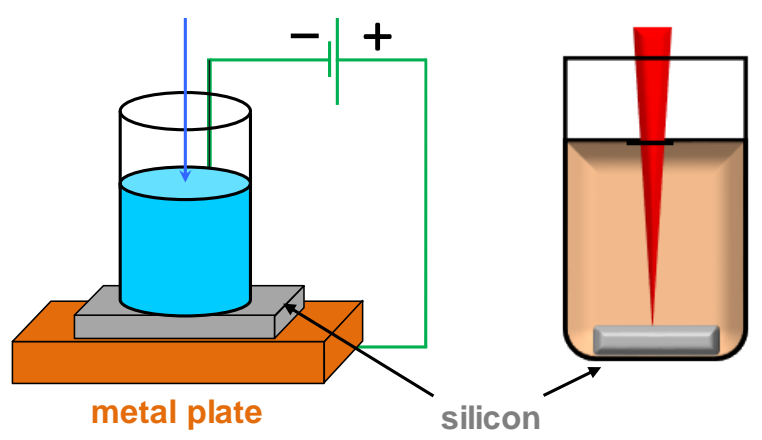

Figure 1. Methods of preparation of silicon nanostructures.

a gold target immersed in the Si NP colloidal solution. In all cases, similar silicon wafers are used (p-type, (100), $10 \Omega \cdot \mathrm{cm}$ ) and treatment time (30 minutes) is also the same.

Size distribution of Si-based NPs is studied using transmission electron microscope (TEM) coupled with energy-dispersive $X$ ray (EDX) spectrometer in order to estimate chemical composition of Si@Au NPs. Experiments on dissolution of Si NPs in the physiological solution $(0.9 \% \mathrm{NaCl}$, volume ratio $1: 1)$ are performed in the dark at room temperature using a dialysis vessel introduced into $5 \mathrm{~L}$ of deionized water. Change of size of NPs is studied by TEM. Optical properties of Si-based NPs are studied using a Shimadzu UV-2700 spectrophotometer (extinction spectra) and a spectrometer based on Solar TII monochromator coupled with nitrogen-cooled CCD camera S7031-1007 Hamamatsu (luminescent properties). Excitation of linear luminescence of Si-based NPs is performed using a nitrogen laser $\left(3.68 \mathrm{eV}, 10 \mathrm{~ns}, 0.2 \mathrm{~mJ} / \mathrm{cm}^{2}\right)$. In order to induce nonlinear optical processes (SHG - second harmonic generation, TPEL - two-photon excited luminescence), a $\mathrm{Nd}$ :YAG laser $\left(1.17 \mathrm{eV}, 10 \mathrm{~ns}, 0.2 \mathrm{~mJ} / \mathrm{cm}^{2}\right)$ is used. Surfaceenhanced Raman scattering (SERS) signal of Rhodamine $B$ molecules is detected by a Raman spectrometer using Si@Au NPs. This spectrometer is based on an Olympus microscope and a cw laser. The used excitation wavelength was $785 \mathrm{~nm}$ at 10.5 $\mathrm{mW}$ power at a sample and the integration time was set to 2 seconds. 


\section{RESULTS AND DISCUSSION}

Formed porous silicon reveals a strong broad luminescent spectrum under $3.68 \mathrm{eV}$ laser excitation (Fig. 2). Subsequent adsorption of various gases $\left(\mathrm{NO}_{2} ; \mathrm{C}_{5} \mathrm{H}_{5} \mathrm{~N} ; \mathrm{NH}_{3} ; \mathrm{O}_{2}\right)$ at different molecule pressures (0.1 - 760 Torr) provokes strong decrease of PL intensity (Figs. 2a and 2b). Moreover, it often leads to either blue or red shift of maximum of a spectral position that differs for various gases. Additionally, changes of full width at
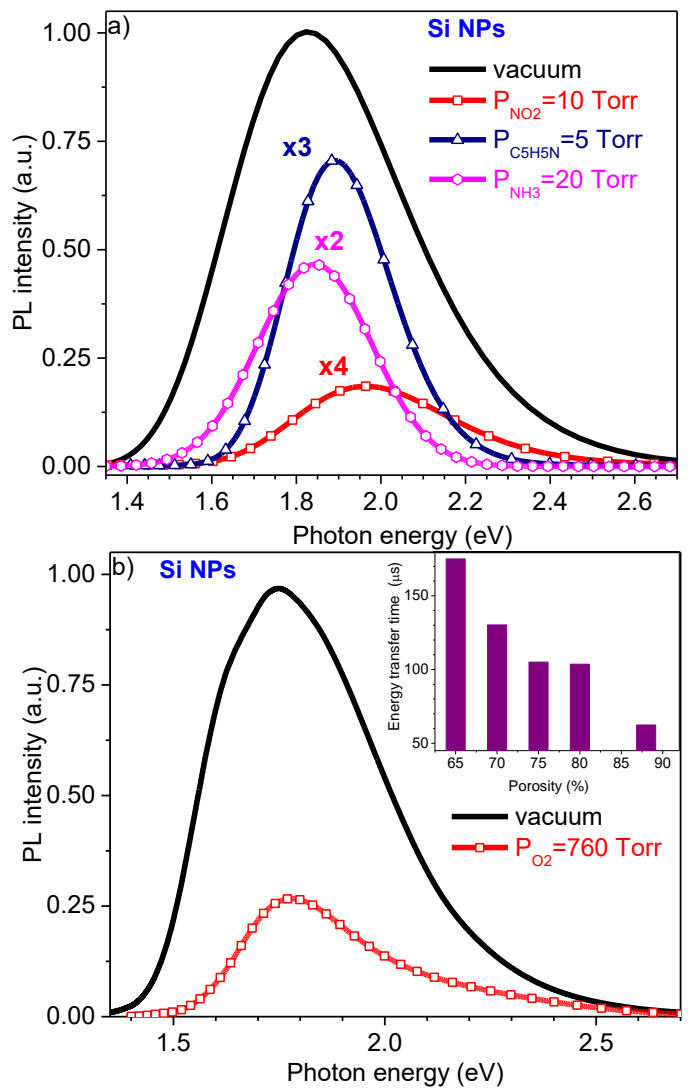

Figure 2. a) Gas detection and b) singlet oxygen generation using electrochemically-prepared silicon nanoparticles.

half maximum (FWHM) can also occur.

It is known that electrochemical etching of a silicon wafer leads to formation of the porous structure due to material removal [Foll 2000; Smith 1992; Lehman 1991]. Structure and properties of formed nanocrystals and surrounding pores strongly depend on wafer properties as well as on etching parameters (electrolyte, current density and etching time). Under the conditions mentioned in the previous section, size of formed nanostructures varies within $5 \mathrm{~nm}$. Such a small size leads to a strong localisation of photo-excited charge carriers provoking formation of excitons with size-dependent electronic properties [Buuren 1998; Hill 1995]. Indeed, the smaller nanocrystals with a stronger localisation of excitons provide a photoluminescent response at higher photon energies [Ledoux 2000; Soni 1999; Hill 1995]. Hence, nanocrystals of different sizes exhibit light at different photon energies resulting in a wide PL spectrum. Its intensity is a result of competition between radiative and nonradiative recombination processes occurring in nanocrystals. It worth noting that such a competition strongly manifests itself in temperature-dependent PL transients that can be fitted by the stretched exponential decay [Dovrat 2004; Kanemitsu 1996; Pavesi 1993].

It is known that porous silicon has a wide specific surface area ( $\sim 800 \mathrm{~m}^{2} / \mathrm{g}$ ) as compared to monocrystalline wafers [Wongmanerod 2001; Herino 2000; Halimaoui 1994]. This leads to a considerable sensitivity of its photoluminescence to molecular environment. Indeed, adsorption of all gases leads to decrease of PL intensity. In the case of $\mathrm{NO}_{2}, \mathrm{C}_{5} \mathrm{H}_{5} \mathrm{~N}, \mathrm{NH}_{3}$, adsorption on the surface of silicon nanocrystals leads to formation of positively or negatively charged complexes and subsequent change of concentration of defect states that can be detected by electron paramagnetic resonance (EPR) spectroscopy [Osminkina 2015; Kashkarov 2007; Konstantinova 2005; Pavlikov 2005; Sharov 2005; Skryshevsky 2000]. Strong local electric field provided by these complexes considerably influences exciton behaviour leading to destruction of excitons and subsequent nonradiative recombination of photoexcited charge carriers. Evidently, PL quenching is more pronounced in larger Si nanocrystals with lower binding energy of excitons. Hence, it leads to experimentally observed blue shift of PL spectra (nitrogen dioxide, pyridine, ammonia) and change of PL intensity that can be used for sensing of dangerous gases [Sharov 2005; Konstantinova 2004; Baratto 2001; Harper 1996]. An additional mechanism of PL changes is related to oxidation of surface of silicon nanocrystals in oxygen-containing atmosphere.

In the case of oxygen molecules, an additional mechanism related to Förster resonant energy transfer (FRET) can also play an important role remarkably influencing PL behaviour of silicon nanostructures. It has been shown that effective generation of singlet oxygen can be provoked by external sensitizers like dye molecules [Awuah 2011, Adarsh 2010; Shi 2006; DeRosa 2002]. Moreover, silicon nanostructures can also play role of effective photosensitizers of singlet oxygen generation. Indeed, being adsorbed on the surface of silicon nanostructures, they ensure effective energy transfer from photoexcited charge carriers in silicon nanocrystals to oxygen molecules, transforming them to the excited singlet state [Osminkina 2011; Xiao 2011; Fuji 2006; Timoshenko 2006; Kovalev 2005; Fuji 2004; Kovalev 2004]. As a result, this leads to a significant reduction of a PL signal of porous silicon nanostructures. It is worth noting that the most effective generation of singlet oxygen (the most pronounced PL quenching) is detected at $760 \mathrm{~nm}$ that corresponds to the energy transfer between triplet and singlet states of oxygen molecules.

Interestingly, silicon nanoparticles prepared by laser ablation of a silicon wafer show a completely different luminescent behaviour. Indeed, one can see that excitation of LA-Si NPs at $3.68 \mathrm{eV}$ doesn't lead to any detectable emission response (Fig. 3a). However, their excitation using an IR laser (1.17 eV) allows detection of a remarkable optical signal (Fig. 3a). Its efficiency considerably depends on the excitation level as well as on laser wavelength. Moreover, size of LA-Si NPs also influences efficiency of their non-linear properties [Kharin 2019]. It worth noting that emission range $(\sim 1.5-1.8 \mathrm{eV})$ is similar to that of PSSi NPs.

Absence of any linear-excited photoluminescence can be caused by different reasons. Firstly, laser-synthesized Si NPs possess large mean size of formed nanocrystals ( $45 \mathrm{~nm})$ as it can be seen in Fig. 3b [Ryabchikov 2019a]. In this case, binding energy of excitons is too low and they cannot be stable at room temperature. Secondly, laser ablation provokes large amount of defects states as it can be stated from EPR measurements [Ryabchikov 2019a]. Performed analysis reveals that this type of paramagnetic defects corresponds to silicon dangling bonds in disordered silicon [Baran 2004; Bardeleben 1993; Shimasaki 1996]. Hence, it can be an additional mechanism of nonradiative recombination of photoexcited charge carriers leading to the absence of linear luminescent response. The nonlinear optical properties of Si NPs can be explained taking 

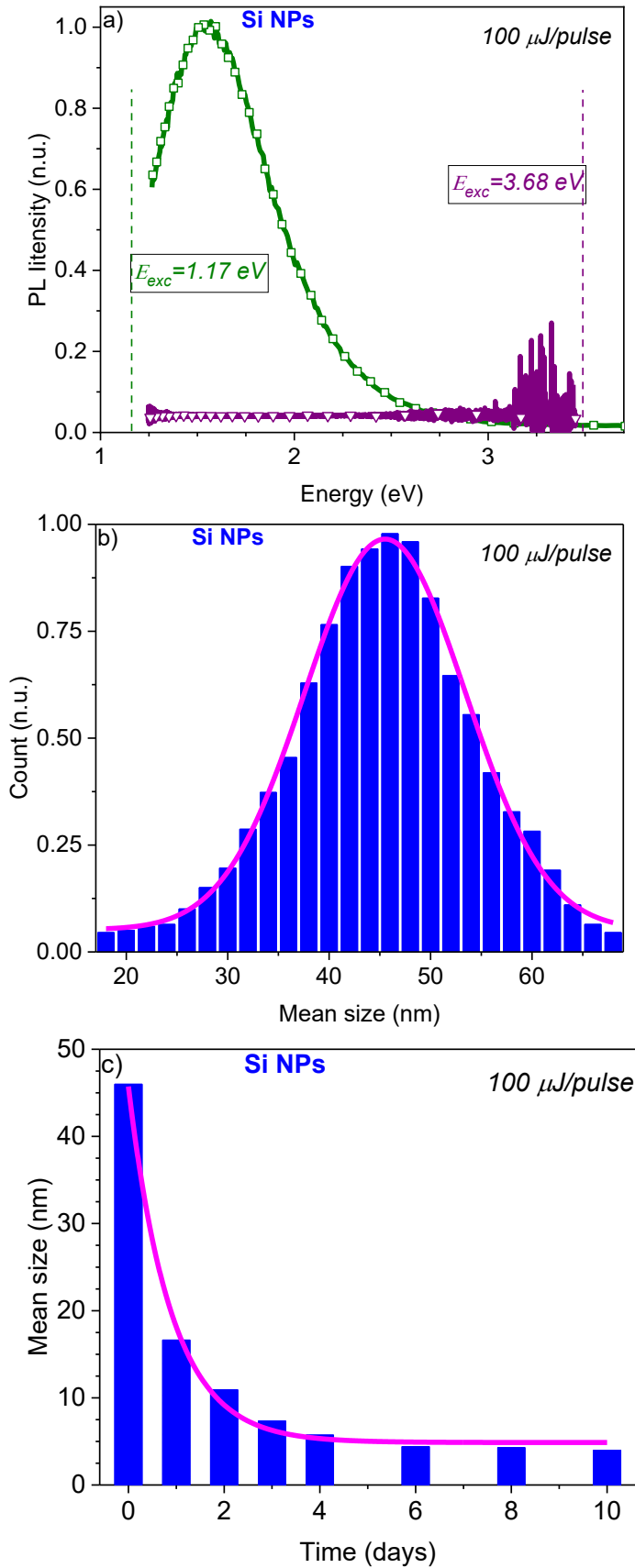

Figure 3. Properties of laser-synthesized Si NPs: a) linear and non-linear photoluminescence ( $E_{\mathrm{exc}}=1.17 \mathrm{eV}$ and $3.68 \mathrm{eV}$ ), b) size distribution, c) time-dependent dissolution behaviour nanoparticles. Laser fluence used for laser ablation is $\mathbf{1 0 0}$ $\mu \mathrm{J} /$ pulse.

into account 2-photon excitation of charge carriers in the core of NPs followed by their trapping and radiative recombination at $\mathrm{Si} / \mathrm{SiO}_{2}$ interface as well as direct excitation of electronic defect states in $\mathrm{SiO}_{2}$ shell due to 2-photon absorption [Kharin 2019]. It worth noting that besides TPEL, laser-synthesized Si NPs also reveal quite strong SHG signal that is shown to be very promising for bioimaging applications [Kharin 2019].

As shown above (Fig. 2a, 2b), properties of silicon nanoparticles may also be strongly affected by surrounding media. Indeed, study of the behaviour of LA-Si NPs in a biological medium shows their fast dissolution due to size degradation (Fig. 3c).
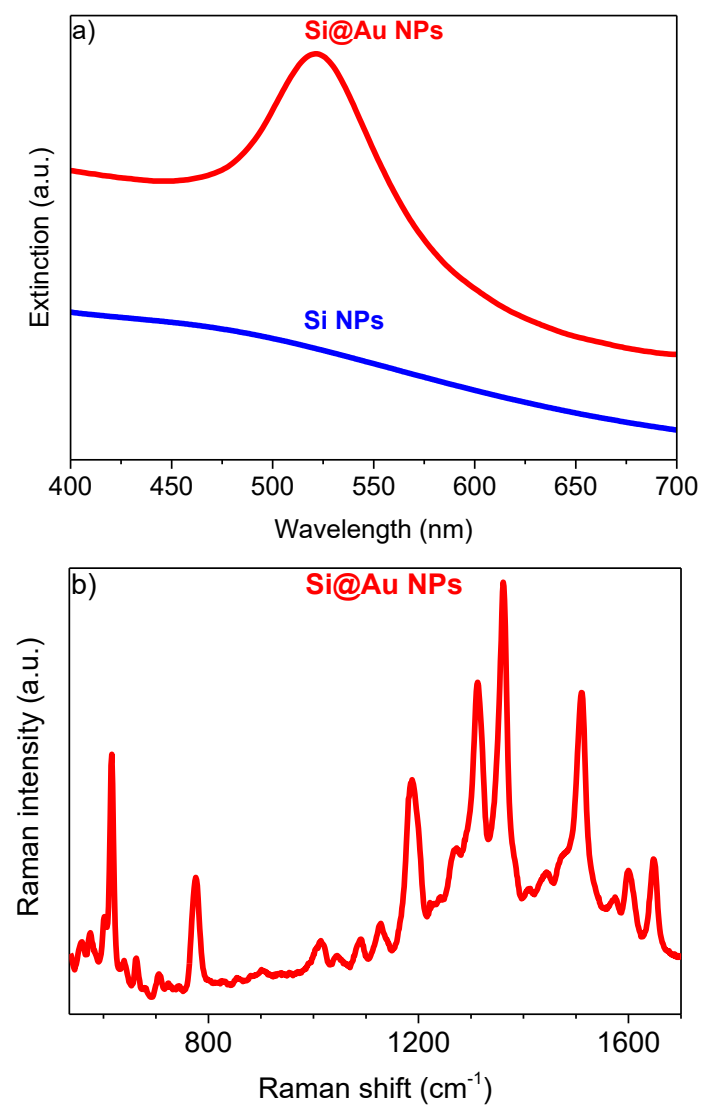

Figure 4. a) Extinction spectra of Si and Si@Au NPs, b) Raman spectra of Rhodamine B using surface enhancement by Si@Au NPs.

One can see that, during first 2-3 days, their size is decreased by a factor of 4 [Ryabchikov 2019b; Al-Kattan 2016]. It worth noting that PS-Si NPs show a fast dissolution dynamics offering their perspectives for in-vivo applications [Park 2009]. The dissolution is determined by interaction of laser-formed Si NPs with oxygen dissolved in water that provokes further oxidation of Si core. It leads to transformation of Si NPs into orthosilic acid $\mathrm{Si}(\mathrm{OH})_{4}$ that can be naturally excreted from an organic with urine [Ksenofontova 2014]. Hence, this important property makes LA-Si NPs very promising for biological applications due to the possibility of their fast and easy extraction from an organism after their diagnostic or therapeutic actions.

As any semiconductor nanomaterials, LA-Si NPs manifest such a limitation as the absence of plasmonic properties (Fig. 4a), which are associated with metal-based nanostructures used for any plasmonic-related applications [Bansal 2015; Ding 2016; Li 2016; Nugroho 2016; Singh 2017; Yang 2016; Ye 2017]. In order to overcome this lack, Si NPs can be structurally modified using recently developed method based on laser ablation [Ryabchikov 2019]. It has been found that it leads to a remarkable change of optical properties of formed Si@Au NPs due to incorporation of some metal species. As a result, considerable plasmonic maximum appears at around $515 \mathrm{~nm}$ corresponding to nanostructured gold (Fig. 4a). It significantly enhances functionality of LA-Si NPs toward detection of biological systems using surface-enhanced optical response, in particular, SERS or SEIRA (surface-enhanced infrared absorption) [Ryabchikov 2019b; Kogler 2018; Bibikova 2017]. In order to show ability of Si@Au NPs for SERS purpose, highly Raman-active dye molecules are used. Their concentration is chosen to be undetectable by Raman spectroscopy without any external sensitizers. 

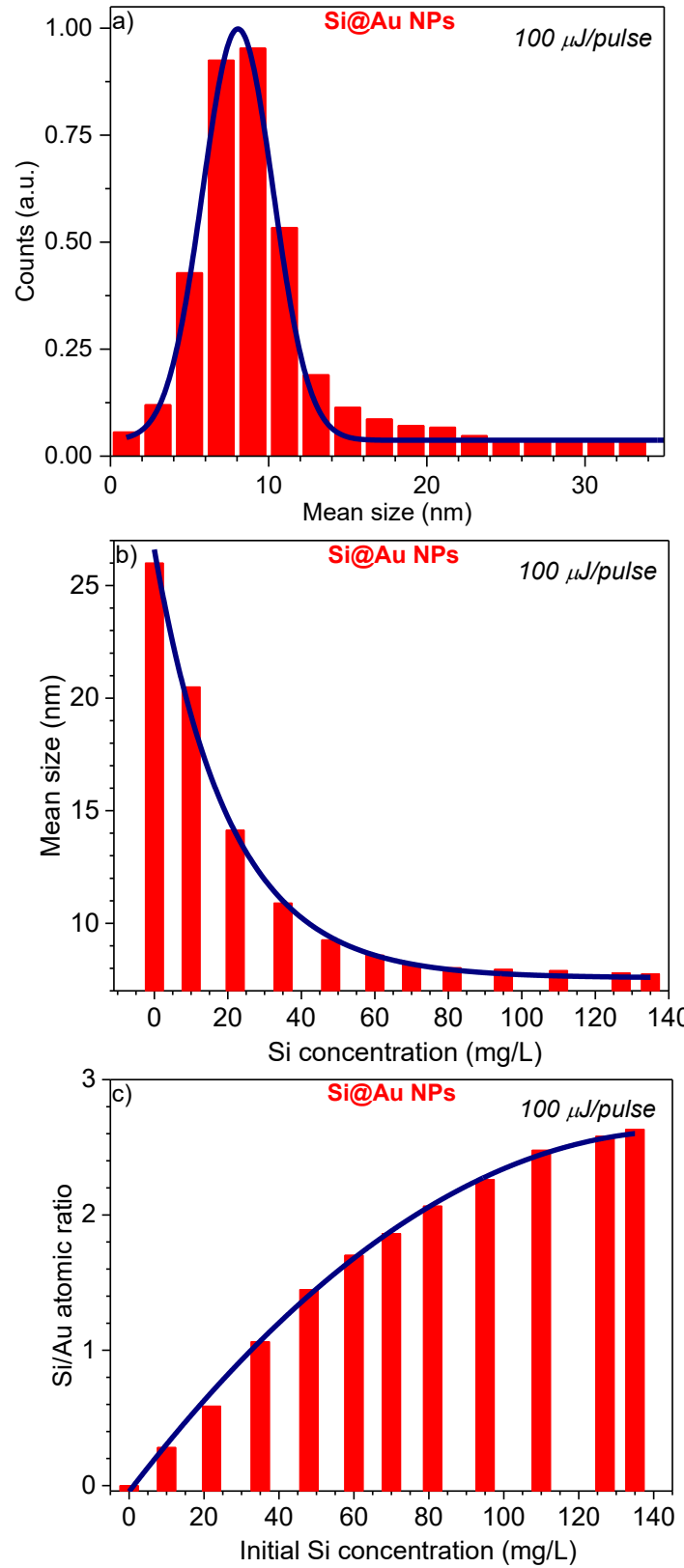

Figure 5. Properties of laser-synthesized Si@Au NPs: a) size distribution, b) concentration-dependent mean size, c) concentration-dependent chemical composition. Laser fluence used for laser ablation is $100 \mu \mathrm{J} /$ pulse.

In Fig. 4b, one can see that rhodamine $B$ molecules can be easily detected using SERS when combined with Si@Au NPs [Ryabchikov 2019b; Kogler 2018]. Such a capability of composite Si-based NPs is conditioned by plasmonic properties due to laser-induced structural modification.

As it is mentioned above, laser ablation of a gold target immersed in colloidal solution of LA-Si NPs leads to considerable structural changes of the latter. It can be seen in Fig. 5 in more details. First of all, such a process significantly changes the mean size of newly formed composite NPs (Fig. 5a). Moreover, it can be stated that their distribution is also significantly reduced compared to initial pure $\mathrm{Si} N P s$ [Ryabchikov 2019a]. It should be noted that the initial concentration of Si NP colloidal solution significantly affects both mean size and chemical composition of Si@Au NPs (Figs. $5 b, 5 c)$. One can see that a larger amount of Si NPs in the initial solution remarkably decreases the mean size of the composite nanoparticles. After some threshold ( 100 mg/L), size of Si@Au
NPs is independent on the initial concentration of Si NP colloid (Fig. 5b). At the same time, large concentration of nanostructured silicon also increases its content in the formed composite NPs (Fig. 5c).

Such changes are provoked by a strong interaction between $\mathrm{Si}$ and $\mathrm{Au}$ nanoclusters formed due to laser influence on Si NPs and a gold target, respectively. Presence of a second matter in the expanding ablation products can significantly restrict the growth of NPs as already reported in literature [Besner 2009; Sylvestre 2004a; Sylvestre 2004b; Kabashin 2003]

\section{CONCLUSIONS}

Optical properties of silicon nanoparticles differ considerably. It is shown that electrochemical etching leads to the efficient linear-excited photoluminescence while laser ablation provokes strong nonlinear optical response. Porous silicon nanostructures show promising perspectives for gas sensing applications as well as for biomedical applications due to formation of singlet oxygen. Laser ablated silicon nanostructures reveal perspectives for bioimaging application due to strong nonlinear optical properties. Their structural modification in the presence of gold leads to significant reduction of their size distribution accompanied with appearance of remarkable plasmonic properties depending on concentration of the initial Si NP colloidal solution. Strong plasmon properties of Si@Au NPs open up new perspectives of Si-based nanomaterials for molecule detection using ability surface enhancement of optical signals.

\section{ACKNOWLEDGMENTS}

The work is financially supported by the Ministry of Education, Youth and Sports of the Czech Republic (Programmes NPU IProject no. LO1602) and Large Research Infrastructure Project No. LM2015086).

\section{REFERENCES}

[Adarsh 2002] Adarsh, N. et al. Tuning Photosensitized Singlet Oxygen Generation Efficiency of Novel Aza-BODIPY Dyes. Org. Lett., 2010, Vol. 12, No. 24, pp 57205723

[Al-Kattan 2016] Al-Kattan, A. et al. Ultrapure lasersynthesized Si nanoparticles with variable oxidation state for biomedical applications. Journal of Materials Chemistry B, 2016, Vol. 4, pp 7852-7858

[Awuah 2011] Awuah, S.G. et al. Singlet Oxygen Generation by Novel NIR BODIPY Dyes. Org. Lett., 2011, Vol. 13, No. 15 , pp 3884-3887

[Bansal 2015] Bansal, A. and Verma. S.S. Optical response of noble metal alloy nanostructures. Physics Letters A, 2015, Vol. 379, No. 3,pp 163-169

[Baran 2004] Baran, M. et al. Luminescence and EPR studies of defects in $\mathrm{Si}_{-} \mathrm{SiO}_{2}$ films. Eur. Phys. J. Appl. Phys., 2004, Vol. 27, pp 285-287

[Baratto 2001] Baratto, C. A novel porous silicon sensor for detection of sub-ppm $\mathrm{NO}_{2}$ concentrations. Sensors and Actuators B, 2001, Vol. 77, pp 62-66

[Bardeleben 1993] Bardeleben, H.J. et al. Defect and structure analysis of $n+-, p+-$ and $p$-type porous silicon by the electron paramagnetic resonance technique. J. Lumin., 1993, Vol. 57, No. 1-6, pp 301-303

[Besner 2009] Besner, S. et al. Synthesis of Size-Tunable Polymer-Protected Gold Nanoparticles by Femtosecond Laser-Based Ablation and Seed 
Growth. J. Phys. Chem. C, 2009, Vol. 113, No. 22, pp 9526-9531

[Bibikova 2017] Bibikova, O. et al. Surface enhanced infrared absorption spectroscopy based on gold nanostars and spherical nanoparticles. Analytica Chimica Acta, 2017, Vol. 990, pp 141-149

[Brus 1994] Brus, L. Luminescence of Silicon Materials: Chains, Sheets, Nanocrystals, Nanowires, Microcrystals, and Porous Silicon. J. Phys. Chem., 1994, Vol. 98, No. 14, pp 3575-3581

[Buuren 1998] Buuren, T. et al. Changes in the Electronic Properties of Si Nanocrystals as a Function of Particle Size. Phys. Rev. Lett., 1998, vol. 80, No. 17, pp 3803-3806

[Clemens 1997] Clemens, J.T. Silicon microelectronics technology. Bell Labs Technical Journal, 1997, Vol. 2, No. 4, pp 76-102

[Cullis 1997] Cullis, A.G. The structural and luminescence properties of porous silicon. Journal of Applied Physics, 1997, Vol. 82, p 909

[DeRosa 2002] DeRosa, M.C. et al. Photosensitized singlet oxygen and its applications. Coordination Chemistry Reviews, 2002, Vol. 233-234, pp 351-371

[Ding 2016] Ding, S.-Y. et al. Nanostructure-based plasmonenhanced Raman spectroscopy for surface analysis of materials. Nature Reviews Materials, 2016, Vol. 1, pp 1-16

[Dovrat 2004] Dovrat, M. Radiative versus nonradiative decay processes in silicon nanocrystals probed by timeresolved photoluminescence spectroscopy. Phys. Rev. B, 2004, Vol. 9, p 155311

[Foll 2000] Foll, H. Formation and application of porous silicon. Materials Science and Engineering, 2000, Vol. R39, pp 93-141

[Fuji 2006] Fuji, M. et al. Dynamics of photosensitized formation of singlet oxygen by porous silicon in aqueous solution. Journal of Applied Physics, 2006, Vol. 100, p 124302

[Fuji 2004] Fuji, M. et al. Chemical reaction mediated by excited states of Si nanocrystals-Singlet oxygen formation in solution. Journal of Applied Physics, 2004, Vol. 95, p 3689

[Genc 2017] Genc, A. et al. Hollow metal nanostructures for enhanced plasmonics: synthesis, local plasmonic properties and applications. Nanophotonics, 2017, Vol. 6, No. 1, pp 193-213

[Govoni 2012] Govoni, M. et al. Carrier multiplication between interacting nanocrystals for fostering silicon-based photovoltaics. Nature Photonics, 2012, Vol. 6, pp 672-679

[Harper 1996] Harper, J. and Sailor, M.J. Detection of Nitric Oxide and Nitrogen Dioxide with Photoluminescent Porous Silicon. Anal. Chem., 1996, Vol. 68, pp 37133717

[Halimaoui 1994] Halimaoui, A. Determination of the specific surface area of porous silicon from its etch rate in HF solutions. Surface Science, 1994, Vol. 306, No. 12, pp L550-L554

[Heinrich 1992] Heinrich, J.L. Luminescent Colloidal Silicon Suspensions from Porous Silicon. Science, 1992, Vol. 255, No. 5040, pp. 66-68

[Herino 2000] Herino, R. eta I. Porosity and Pore Size Distributions of Porous Silicon Layers. J. Electrochem. Soc., 1987, Vol. 134, No. 8, pp 19942000
[Hill 1995] Hill, N.A. and Whaley, K.B. Size Dependence of Excitons in Silicon Nanocrystals. Phys. Rev. Let., 1995, Vol. 75, No. 6, pp 1130-1133

[Intartaglia 201] Intartaglia, R. et al. Optical Properties of Femtosecond Laser-Synthesized Silicon Nanoparticles in Deionized Water. J. Phys. Chem. C, 2011, Vol. 115, pp 5102-5107

[Kabashin 2003] Kabashin, A.V. et al. Fabrication and Characterization of Gold Nanoparticles by Femtosecond Laser Ablation in an Aqueous Solution of Cyclodextrins. J. Phys. Chem. B, 2003, Vol. 107, No. 19, pp 4527-4531

[Kashkarov 2007] Kashkarov, P.K. et al. Control of charge carrier density in mesoporous silicon by adsorption of active molecules. Physica Status Solidi A, 2007, Vol. 204, No. 5, pp 1404-1407

[Kharin 2019] Kharin, A.Yu. et al. Bi-modal nonlinear optical contrast from $\mathrm{Si}$ nanoparticles for cancer theranostics. Advanced Optical Materials, 2019, Vol. 7, No. 13, p 18011728

[Kogler 2018] Kogler M. at al. Bare Laser-Synthesized AuBased Nanoparticles as Non-Disturbing SERS Probes for Bacteria Identification. Journal of Biophotonics, 2018, Vol. 11, No. 7, p e201700225

[Ledoux 2000] Ledoux, G. et al. Photoluminescence properties of silicon nanocrystals as a function of their size. Phys. Rev. B, 2000, Vol. 62, No. 23, pp 15942-15951

[Lehmann 1991] Lehmann, V. and Gösele. U. Porous silicon formation: A quantum wire effect. Appl. Phys. Lett., 1991, Vol. 58, pp 856-858

[Li 2016] Li, X. et al. Hybrid nanostructures of metal/twodimensional nanomaterials for plasmon-enhanced applications. Chem. Soc. Rev., 2016, Vol. 45, pp 3145-3187

[Kanemitsu 1996] Kanemitsu, Y. et al. Photoluminescence spectrum and dynamics in oxidized silicon nanocrystals: A nanoscopic disorder system. Phys. Rev. B, 1996, Vol. 53, No. 20, pp 13515-13520

[Konstantinova 2005] Konstantinova, E.A. et al. Influence of $\mathrm{NO}_{2}$ molecule adsorption on free charge carriers and spin centers in porous silicon. Physica Status Solidi A, 2005, Vol. 202, No. 8, pp 1592-1596

[Konstantinova 2004] Konstantinova, E.A. et al. Effect of Adsorption of the Donor and Acceptor Molecules at the Surface of Porous Silicon on the Recombination Properties of Silicon Nanocrystals. Semiconductors, 2004, Vol. 38, No. 11, pp 1344-1349

[Kovalev 2005] Kovalev, D. and Fuji. M. Silicon Nanocrystals: Photosensitizers for Oxygen Molecules. Advanced Materials, 2005, Vol. 17, No. 21, pp 2531-2544

[Kovalev 2004] Kovalev, D. et al. Photodegradation of porous silicon induced by photogenerated singlet oxygen molecules. Appl. Phys. Lett., 2004, Vol. 85, p 3590

[Ksenofontova 2014] Ksenofontova, O.I., et al. Porous silicon and its applications in biology and medicine. Technical Physics, 2014, Vol. 59, No. 1, pp 66-77.

[Kuzmin 2010] Kuzmin, P.G. et al. Silicon Nanoparticles Produced by Femtosecond Laser Ablation in Ethanol: Size Control, Structural Characterization, and Optical Properties. J. Phys. Chem. C, 2010, Vol. 114, pp 15266-15273

[Nugroho 2016] Nugroho, F.A.A. et al. Bottom-Up Nanofabrication of Supported Noble Metal Alloy Nanoparticle Arrays for Plasmonics. ACS Nano, 2016, Vol. 10, No. 2, pp 2871-2879 
[Osminkina 2015] Osminkina, L.A. et al. The role of boron impurity in the activation of free charge carriers in layers of porous silicon during the adsorption of acceptor molecules. Semiconductors, 2015, Vol. 39, No. 3, pp 347-350

[Osminkina 2011] Osminkina, L.A. et al. Silicon nanocrystals as photo- and sono-sensitizers for biomedical applications. Applied Physics B, 2011, Vol. 105, No. 3, pp 665-668

[Park 2009] Park, J.-H. Biodegradable luminescent porous silicon nanoparticles for in vivo applications. Nature Materials, 2009, Vol. 8, pp 331-336

[Pavlikov 2005] Pavlikov, A.V. et al. Effect of the initial doping level on changes in the free-carrier concentration in porous silicon during ammonia adsorption. Semiconductors, 2005, Vol. 39, No. 11, pp 13381341

[Qin 1993] Qin, G.G. and Jia, Y.Q. Mechanism of the visible luminescence in porous silicon. Solid State Communications, 1993, Vol. 86, No. 9, pp 559-563

[Pavesi 1993] Pavesi, L. and Ceschini, M. Stretched-exponential decay of the luminescence in porous silicon. Phys. Rev. B, 1993, Vol. 48, No. 23, pp 17625-17628

[Priolo 2014] Priolo, F. et al. Silicon nanostructures for photonics and photovoltaics. Nature Nanotechnology, 2014, Vol. 9, pp 19-32

[Rioux 2009] Rioux, D. et al. Silicon nanoparticles produced by femtosecond laser ablation in water as novel contamination-free photosensitizers. Journal of Biomedical Optics, 2009, Vol. 14, No. 2, p 021010

[Ryabchikov 2019a] Ryabchikov, Yu.V. Size Modification of Optically Active Contamination-Free Silicon Nanoparticles with Paramagnetic Defects by Their Fast Synthesis and Dissolution. Physica Status Solidi, 2019, Vol. 216, No. 2, p A1800685

[Ryabchikov 2019b] Ryabchikov, Yu.V. Facile Laser Synthesis of Multimodal Composite Silicon/Gold Nanoparticles with Variable Chemical Composition. Journal of Nanoparticle Research, 2019, Vol. 21, No. 4, p 85

[Semaltianos 2010] Semaltianos, N.G. et al. Silicon nanoparticles generated by femtosecond laser ablation in a liquid environment. J. Nanopart. Res., 2010, Vol. 12, pp 573-580

[Sharov 2005] Sharov, C.S. et al. Chemical Modification of a Porous Silicon Surface Induced by Nitrogen Dioxide Adsorption. J. Phys. Chem. B, 2005, Vol. 109, No. 10, pp 4684-4693

[Shi 2006] Shi L. et al. Singlet Oxygen Generation from WaterSoluble Quantum Dot-Organic Dye Nanocomposites. J. Am. Chem. Soc., 2006, Vol. 128, No. 19, pp 6278-6279

[Shimasaki 1996] Shimasaki, M. et al. Correlation between light emission and dangling bonds in porous silicon. Appl. Surf. Sci., 1996, Vol. 92, pp 617-620

[Singh 2017] Singh, J. Structural, Optical and Plasmonic Properties of $\mathrm{Ag}-\mathrm{TiO} 2$ Hybrid Plasmonic Nanostructures with Enhanced Photocatalytic
Activity. Plasmonics, 2017, Vol. 12, No. 3, pp 877 888

[Skryshevsky 2000] Skryshevsky, V.A. Photoluminescence of inhomogeneous porous silicon at gas adsorption. Applied Surface Science, 2000, Vol. 157, No. 3, pp $145-150$

[Smith 1992] Smith, R.L. and Collins, S.D. Porous silicon formation mechanisms. J. Appl. Phys., 1992, Vol. 71, pp. R1-R22

[Soni 1999] Soni, R.K. et al. Size-dependent optical properties of silicon nanocrystals. Journal of Luminescence, 1999, Vol. 83-84, pp 187-191

[Sylvestre 2004a] Sylvestre, J.-P. et al. Stabilization and Size Control of Gold Nanoparticles during Laser Ablation in Aqueous Cyclodextrins. J. Am. Chem. Soc., 2004a, Vol. 12, No. 62, pp 37176-7177

[Sylvestre 2004b] Sylvestre, J.-P. et al. Surface Chemistry of Gold Nanoparticles Produced by Laser Ablation in Aqueous Media. J. Phys. Chem. B, 2004b, Vol. 108, No. 43, pp 16864-16869

[Timoshenko 2006] Timoshenko, V. Yu. et al. Silicon nanocrystals as photosensitizers of active oxygen for biomedical applications. JETP Letters, 2006, Vol. 83, No. 9, pp 423-426

[Tischler 1992] Tischler, M.A. Luminescence degradation in porous silicon. Appl. Phys. Lett., 1992, Vol. 60, p 639

[Vetterl 2000] Vetterl, O. et al. F. Intrinsic microcrystalline silicon: A new material for photovoltaics. Solar Energy Materials and Solar Cells, 2000, Vol. 62, No. $1-2$, pp 97-108

[Wongmanerod 2001] Wongmanerod, C. et al. Determination of pore size distribution and surface area of thin porous silicon layers by spectroscopic ellipsometry. Applied Surface Science, 2001, Vol. 172, No. 1-2, pp 117-125

[Xiao 2011] Xiao, L. et al. Porous Silicon Nanoparticle Photosensitizers for Singlet Oxygen and Their Phototoxicity against Cancer Cells. ACS Nano, 2011, Vol. 55, pp 3651-3659

[Xie 1992] Xie, Y.H. Luminescence and structural study of porous silicon films. Journal of Applied Physics, 1992, Vol. 71, p 2403

[Yang 2016] Yang, P. et al. Colloidal Synthesis and Applications of Plasmonic Metal Nanoparticles. Adv.Mater., 2016, Vol. 28, pp 10508-10517

[Yates 1998] Yates, J.T. A New Opportunity in Silicon-Based Microelectronics. Science, 1998, Vol. 279, No. 5349, pp 335-336

[Ye 2017] Ye, W. et al. Plasmonic nanostructures in solar energy conversion. J. Mater. Chem. C, 2017, Vol. 5, pp 1008-1021

[Yue 2014] Yue, D. et al. Domestic and overseas manufacturing scenarios of silicon-based photovoltaics: Life cycle energy and environmental comparative analysis. Solar Energy, 2014, Vol. 105, pp 669-678

\section{CONTACTS:}

Dr. Yury Ryabchikov

HiLASE Centre of Institute of Physics of the Czech Academy of Sciences

Za Radnicí 828, Dolní Břežany, 25241, Czech Republic

+420 3140077 34, yury.ryabchikov@hilase.cz,www.hilase.cz 